\title{
Leitthema
}

Hautarzt 2015 - 66:829-833

DOI 10.1007/s00105-015-3676-z

Online publiziert: 7. September 2015

(c) Die Autor(en) 2015. Dieser Artikel ist auf

Springerlink.com mit Open Access verfügbar

CrossMark

\author{
I. Kruglikov \\ Wellcomet GmbH, Karlsruhe, Deutschland
}

\section{Sehr hochfrequenter Ultraschall}

\section{Neues Therapieverfahren in der Ästhetik und Dermatologie}

Die Anzahl von neuen Therapieverfahren in der Dermatologie und besonders in der ästhetischen Medizin ist in den letzten Jahren deutlich gestiegen. Das hat etwas mit der steigenden Nachfrage nach immer mehr zeitsparenden und effizienteren, aber auch finanziell lukrativen Behandlungsmethoden zu tun. Während die meisten dermatologischen Methoden der letzten 2 Jahrzehnte auf Basis der Lichtenergie entstanden sind (basierend auf selektiver Absorption verschiedener Lichtwellenlängen in unterschiedlichen Chromophoren), wurden auch elektromagnetische, thermische und schallbasierende Therapieverfahren immer weiterentwickelt.

Diese Entwicklung ist deutlich am Beispiel der Schallwellen zu sehen. Während noch vor 20 Jahren die Schallwellen in der Dermatologie lediglich in Form von Ultraschall (US) mit Frequenzen von $30-40 \mathrm{kHz}$ (niederfrequenter US) oder $1 \mathrm{MHz}$ (hochfrequenter US) zur Wundheilung $[28,29]$ oder zu Phonophorese [18] eingesetzt wurden, haben sich die Schallwellencharakteristiken und Indikationsspektren in heutigen Applikationen erheblich verändert. So wurden niederfrequente sowie hochintensive fokussierte (HIFU) US-Wellen zur Fettzerstörung eingesetzt. Gleichzeitig wurden hochenergetische fokussierte US-Wellen mit verschiedenen Frequenzen zur Hautfestigung in der ästhetischen Medizin angewendet. Parallel dazu wurde auch der therapeutische Frequenzbereich für USWellen verbreitert und Frequenzen von $10 \mathrm{MHz}$ und höher [sehr hochfrequenter
US (SHF-US)] für Hautbehandlungen eingesetzt.

\section{Kurze physikalische Beschreibung}

Spezielle Wirkungen von SHF-US auf die Haut sind eng mit seinen physikalischen Eigenschaften verbunden. Zum Vergleich: US-Wellen mit Frequenzen von $20 \mathrm{kHz}$, 1 und $10 \mathrm{MHz}$ haben im Wasser jeweils Wellenlängen von $75,1,5$ und $0,15 \mathrm{~mm}$. Bei einer Schallintensität von $1 \mathrm{~W} / \mathrm{cm}^{2}$ erzielen solche Wellen frequenzunabhängige Druckamplituden von bis zu 1,7 bar. Daraus resultiert, dass die erzeugten Druckgradienten (Druckabfall auf der Halbwellenlängedistanz) in einer Schallwelle von $10 \mathrm{MHz} 500$-mal größer sind als in einer US-Welle mit einer Frequenz von $20 \mathrm{kHz}$. Mit steigender Frequenz erhöhen sich die Werte noch weiter. Solche großen lokalen Druckgradienten produzieren mechanische Wirkungen, die bei niedrigeren Frequenzen nicht erzielt werden können.

\author{
SHF-US kann in Form von \\ mono- oder multifrequenten \\ Wellen eingesetzt werden.
}

Bis jetzt wurden in der Praxis nur dualfrequente US-Wellen unter der Bezeichnung LDM $^{\circledast}$ (lokale dynamische Mikromassage) mit sehr schnellem (innerhalb von Mikro- oder Millisekunden) Frequenzwechsel eingesetzt (• Abb. 1; [11, 14]). In solchen Wellen variieren die lokalen Druckgradienten im Gewebe im Takt des Frequenzwechsels, was zusätzlich zu einem speziellen Mikromassageeffekt im Inneren des Gewebes führt.

\section{Besondere Eigenschaften des sehr hochfrequenten Ultraschalls}

Es gibt mindestens 2 Gründe, warum der SHF-US bei dermatologischen und ästhetischen Anwendungen interessant sein kann:

- starke Frequenzabhängigkeit der Eindringtiefe der US-Welle,

- besondere biophysikalische Wirkungen von SHF-US.
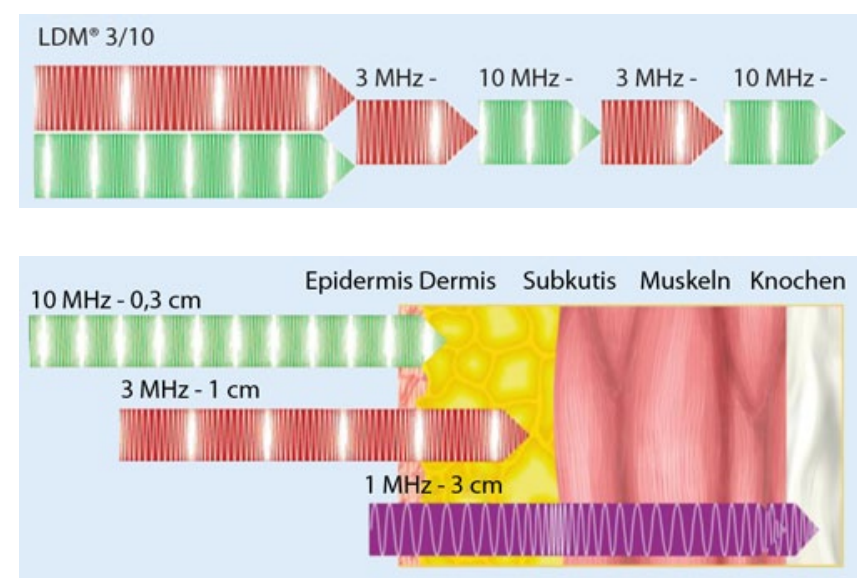

Abb. $1<$ Frequenzwechsel in der LDM $^{\circledast}$ (lokale dynamische Mikromassage)-Technologie

Abb. $2 \triangleleft$ Eindringtiefen von US-Wellen mit verschiedenen Frequenzen 


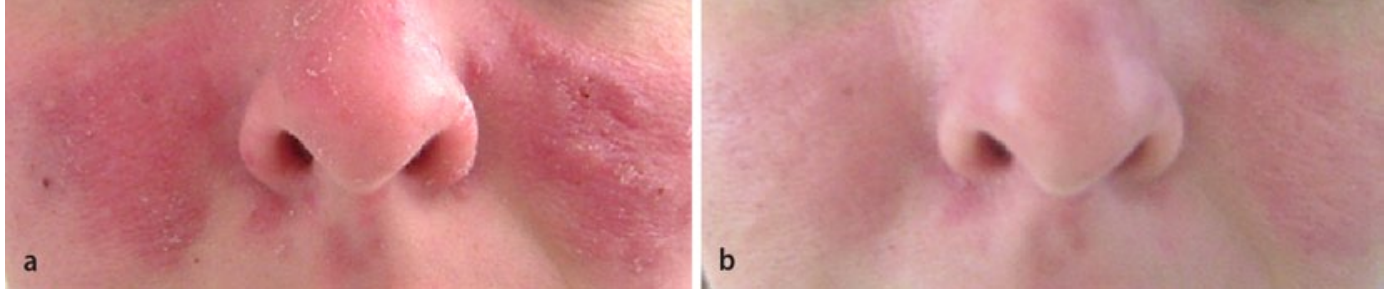

Abb. $3<$ Hautbildverbesserung bei Patientin mit Akne mit kontralateraler Kontrolle: a vorher, b 3 Wochen nach $5 \mathrm{LDM}^{\circledast}$ Behandlungen
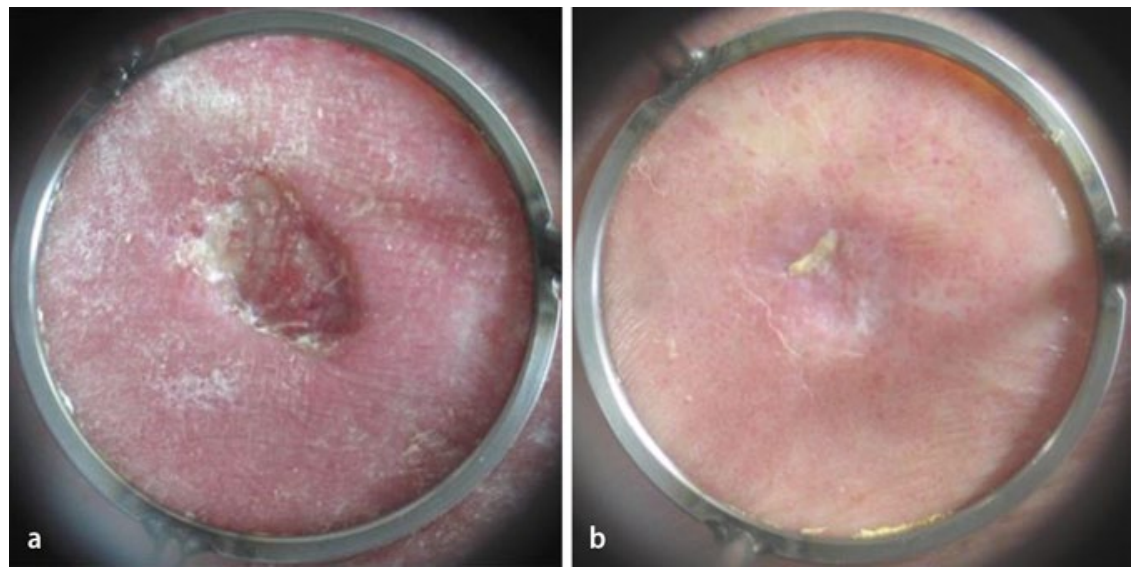

Abb. $4 \Delta$ Chronische (ca. 2 Jahre) Wunde bei Patientin mit diabetischem Fuß: a vorher, b 3 Wochen nach 8 LDM ${ }^{\circledast}$-Behandlungen. (Mit freundl. Genehmigung Scientific Research Publishing)

\section{Eindringtiefe}

Die Eindringtiefe der US-Welle wird mit steigender Frequenz deutlich reduziert (• Abb. 2). Die sog. Halbwerttiefe (die Strecke, nach der sich die Intensität der US-Welle halbiert) bei US-Frequenzen von 1,3 und $10 \mathrm{MHz}$ beträgt beispielweise ca. 3,1 und $0,3 \mathrm{~cm}$. Je höher die USFrequenz von da ab ist, desto oberflächlicher wird die eingeschallte Energie absorbiert. Diese physikalische Eigenschaft ist besonders dann wichtig, wenn eine gewisse Energie bevorzugt in der Dermis absorbiert werden muss, um den gewünschten Effekt zu erzielen. Diese Eigenschaft wird auch bei diagnostischem US berücksichtigt, wo die höheren Frequenzen eingesetzt wurden.

\section{Biophysikalische Wirkungen - präklinische Untersuchungen}

Zelluläre und extrazelluläre Wirkungen der Schallwelle definieren letztendlich die klinischen Ergebnisse. Es wurde allerdings festgestellt, dass die erzielten biophysikalischen Wirkungen nicht nur intensitäts-, sondern auch frequenzabhän- gig sind. Hier werden nur einige Effekte kurz analysiert.

Je nach Frequenz und Intensität, können US-Wellen die Aktivitäten von Matrixmetalloproteinasen (MMP) entweder stimulieren oder reduzieren [11], was eine selektive Anwendung von solchen Wellen bei unterschiedlichen dermatologischen Problemen, wie beispielweise entzündlichen Hauterkrankungen und hypertrophen Narben, vermuten lässt. Tatsächlich ist eine erhöhte Produktion von MMP-1, -3 und -9 bei Akne ein sehr wichtiges pathophysiologisches Merkmal dieser Erkrankung [7], wobei eine Reduktion der MMP-Expression signifikant mit der Hautbildverbesserung korreliert [4]. Eine Überproduktion von MMP-9 durch Keratinozyten ist zumindest teilweise für eine epidermale Entzündung verantwortlich und scheint in die Pathophysiologie der atopischen Dermatitis involviert zu sein [19]. Eine langfristig erhöhte Produktion von MMP-2 und MMP-9 sind bekannte Merkmale von chronischen Wunden [12], wobei besonders MMP-9 für eine verzögerte Wundheilung verantwortlich zu sein scheint [20]. Eine Suppression von MMP2 und eine Stimulation von MMP-9 korre- lieren wesentlich mit einer klinischen Verbesserung von hypertrophen Narben [21]. Dies könnte so interpretiert werden, dass MMP-2 eine "pro-narbenbildende " und MMP-9 eine ,anti-narbenbildende "Wirkung zeigen. Diese wichtigen Ergebnisse wurden durch Tanriverdi-Akhisaroglu et al. [25] bestätigt: Die höchste Konzentration von MMP-2 wurde dabei in Keloiden festgestellt, gefolgt von der Konzentration bei hypertrophen Narben, normaler Haut und atrophischen Narben.

Noch deutlicher wurde diese Frequenzabhängigkeit bei Hitzeschockproteinen (HSP): Bei Anwendung von gleichen Schallintensitäten kann sich z. B. die Aktivierung von Hsp72 bei verschiedenen US-Frequenzen um einige Größenordnungen unterscheiden [24], wobei die SHF-US-Frequenzen von $10 \mathrm{MHz}$ die stärkste und langfristigere Wirkung auf HSPs zeigen. HSPs sind in verschiedene dermale Prozesse involviert [16], z. B. in Wundheilung und Narbenbildung [27]. Hsp72 ist ein molekulares Chaperon, welches für die Proteinkontrolle verantwortlich ist. Seine Expression ist in normal heilenden Wunden stark aktiviert, bleibt aber in chronischen Wunden signifikant unterdrückt [17], was die Modulation von Hsp72 zu einem potenziellem Ziel der Behandlung macht $[1,11]$.

\section{$>$}

Auch Hautalterungsprozesse können durch SHF-US modifiziert werden.

Die überwiegende Konzentration der absorbierten US-Energie in der Dermis führt zu einem moderaten Temperaturanstieg in dieser Hautschicht, welche - wie immer bei milder Hyperthermie - eine lokale Erhöhung von endogenem Hyaluronan zur Folge hat [31]. Diese Ansammlung von Hyaluronan führt zu einer größeren Menge von gebundenem Wasser sowie zur lokalen Erhöhung des Turgors im Bindegewebe. Ähnliche Veränderun- 
gen kann auch die Modulation von MMPund HSP-Expression im Gewebe hervorrufen. Das bildet die Grundlagen für die Anwendung des SHF-US zur Anti-AgingBehandlung $[10,14]$.

Eine wichtige Eigenschaft der US-Wellen ist die Phonophorese (Verstärkung des transdermalen Transports von Wirkstoffen). Phonophorese wurde hauptsächlich für niederfrequente und physiotherapeutische US-Frequenzen untersucht, was auf fehlende klinische Anwendungen von SHF-US zurückzuführen ist. Eine signifikante (wobei auch niedrigere als bei niederfrequentem US) Verstärkung der Phonophorese bei Anwendung des US mit Frequenzen von $10 \mathrm{MHz}$ und größer wurde in $[2,3]$ demonstriert. Trotzdem kann die Anwendung von SHF-US für Phonophorese in der Dermatologie und Ästhetik vorteilhaft sein. Das hat damit zu tun, dass niederfrequenter US in der Haut sog. „lokalisierte Transportregionen“ bildet, die lediglich einen Bruchteil der Hautfläche abdecken. Daraus resultiert ein stark heterogenes fleckenförmiges Hautmuster von Transportzonen. Dieser Effekt tritt bei Anwendung von SHF-US-Wellen nicht auf [18].

Es wurde vor Kurzem demonstriert, dass Fibroblasten, die im Wesentlichen die Strukturen der Dermis bestimmen, durch intradermale Adipozyten aktiviert werden können, was eine große Rolle in Wundheilungsprozessen spielen sollte [23]. Die Anzahl dieser Adipozyten ist von der Proliferation und Differenzierung der adipositären Stammzellen abhängig. US-Wellen zeigen eine deutliche Wirkung auch auf diese Prozesse, wobei auch hier höhere US-Frequenzen deutlich stärkere Effekte hervorrufen [8].

Zusammenfassend kann SHF-US sowohl die Zellen als auch die extrazellulären Strukturen der Haut signifikant beeinflussen, was bei einer frequenzabhängigen Anwendung zu einer gezielten Modifizierung der Dermis und somit zu verschiedenen dermatologisch und ästhetisch relevanten Hautbildveränderungen führen kann.

Zusätzlich steigt die Intensitätsschwellengrenze der Kavitation, die mit der Bildung von freien Radikalen im Gewebe verbunden ist, schnell mit wachsender US-Frequenz an [15]. Dementsprechend

Hautarzt 2015 ·66:829-833 DOI 10.1007/s00105-015-3676-z

(c) Die Autor(en) 2015. Dieser Artikel ist auf Springerlink.com mit Open Access verfügbar

\section{Kruglikov \\ Sehr hochfrequenter Ultraschall. Neues Therapieverfahren in der Ästhetik und Dermatologie}

\section{Zusammenfassung}

Sehr hochfrequenter Ultraschall (SHF-US) stellt ein neues Therapieverfahren mit einem breiten Anwendungsspektrum sowohl in der Dermatologie als auch in der ästhetischen Medizin dar. Dabei werden zu therapeutischen Zwecken Ultraschallwellen mit Frequenzen ab $10 \mathrm{MHz}$ eingesetzt, die bis jetzt nur in der Diagnostik ihre Anwendung gefunden haben. Solche Ultraschallwellen zeigen besondere biophysikalische Wirkungen, was ihren Einsatz $u$. a. bei entzündlichen Hauterkrankungen, chronischen Wunden, hypertrophen Narben sowie zur Anti-Aging-Be- handlung und Hautbildverbesserung ermöglicht. SHF-US kann nicht nur als selbstständige Methode, sondern auch als supportive Behandlung bei der Vor- und Nachsorge von Therapieverfahren wie Laser, radiofrequenten Strömen oder Injektionslipolyse sowie in der ästhetisch-plastischen Chirurgie eingesetzt werden.

Schlüsselwörter

Frequenz · Chronische Wunden · Anti-Aging · Hautbild · Supportive Behandlungsmethode

\section{Very high frequency ultrasound. New therapeutic method in aesthetic medicine and dermatology}

\section{Abstract}

Very high frequency ultrasound (VHF-US) is new therapy method with a broad application spectrum in dermatology and aesthetic medicine. In this method, ultrasound waves with frequencies over $10 \mathrm{MHz}$, which were for a long time only used in ultrasound diagnostics, are applied for therapeutic purposes. Such US waves demonstrate specific biophysical efficiencies which warrant their application for the treatment of the skin efflorescences, chronic wounds and hypertrophic scars as well as in anti-aging and skin im- provement procedures in aesthetic medicine. VHF-US can be applied not only for standalone treatments, but also as a supportive pre- and posttreatment method in combination with laser, radiofrequency currents, injection lipolysis, etc. as well as in aesthetic plastic surgery.

\section{Keywords}

Skin rash · Chronic wounds · Anti-aging . Skin improvement - Supportive treatment methods braucht eine SHF-US-Welle eine viel höhere Intensität als eine konventionelle USWelle, um die Kavitation zu erzielen. Das schließt die Kavitation und ihre potenziellen Nebenwirkungen bei Anwendung von SHF-US fast komplett aus.

\section{Klinische Ergebnisse}

\section{Dermatologie}

Die Wirkung von SHF-US mit $10 \mathrm{MHz}$ auf Akne und Rosazea wurde bereits in einer Pilotstudie geprüft [13]. Die Patienten durften während der Behandlungsphase keine anderen Behandlungsmethoden benutzen. Drei unabhängige Dermatologen ( 2 davon wurden geblindet) führten Bewertungen von Hauteffloreszenzen nach der Leeds-Skala (10 Stufen) durch.
Eine kontralaterale Kontrolle bei 2 Patienten bestätigte eine lokale Wirkung der Methode. Schon nach 1 bis 2 Wochen regulärer Behandlungen mit SHF-US wurde eine durchschnittliche Hautbildverbesserung von über $45 \%$ festgestellt.

Die - Abb. 3 zeigt eine Patientin mit Akne im 1. Trimester der Schwangerschaft, die mit $\mathrm{LDM}^{\circledR}$ im Gesicht behandelt wurde. $\mathrm{LDM}^{\circledR}$ wurde als Behandlungsoption ausgewählt, weil alle Ergebnisse von durchgeführten kontralateralen Behandlungen eine lokale und keine systemische Wirkung dieser Behandlungsmethode gezeigt haben. Die nach 5 Behandlungen erreichte Hautbildverbesserung blieb unverändert bis zum Ende der Schwangerschaft.

Die Anwendung von SHF-US in $\mathrm{LDM}^{\oplus}$-Form wurde unter anderem bei 

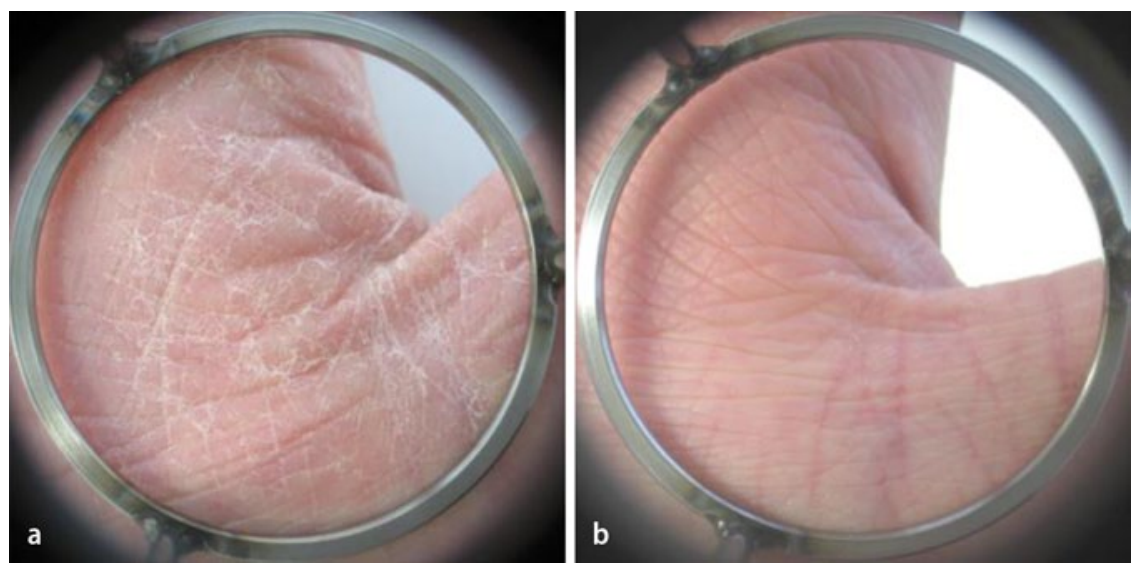

Abb. 5 A Handekzem: a vorher (27.01.2009), b vor der vierten Behandlung (06.02.2009)

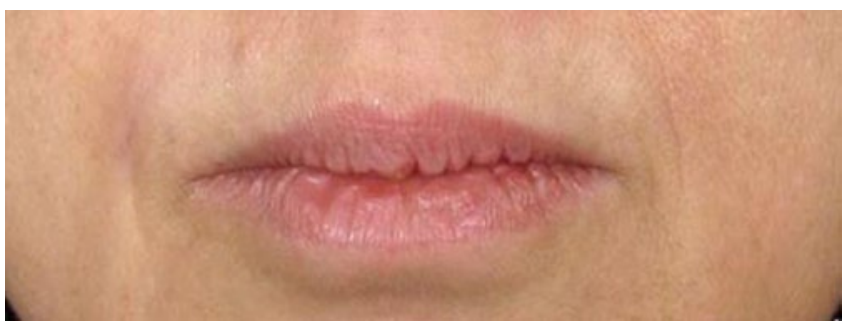

Abb. $6<$ Hautbildverbesserung bei Patientin mit kontralateraler Kontrolle

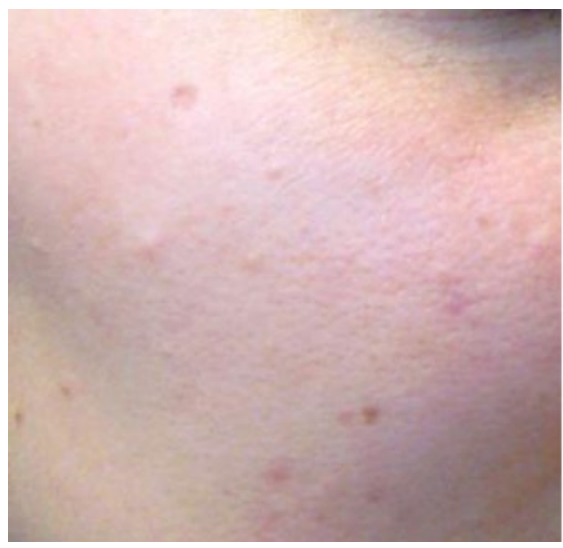

Abb. 7 A Hautbildverbesserung nach 12 Tagen Behandlung mit 10-MHz-SHF-US

chronischen Wunden in einer Pilotstudie an 10 Patienten mit großen $\left(>5 \mathrm{~cm}^{2}\right)$ chronischen ( $>6$ Monate) venösen Ulzera getestet [11]. Alle behandelten Wunden wurden bis zum Ende der Behandlungsserie vollständig reepithelisiert. Follow-up-Untersuchungen ( 3 bis 8 Wochen nach der letzten Behandlung) demonstrierten eine vollständige Abheilung von Wunden. Die - Abb. 4 zeigt ein Beispiel der Wundheilung bei einer 70-jährigen Patientin mit diabetischem Fuß.

Es wurde zusätzlich gezeigt, dass die Anwendung von SHR-US sehr gute Ergebnisse auch bei posttraumatischen, nicht heilenden Wunden [6] sowie bei

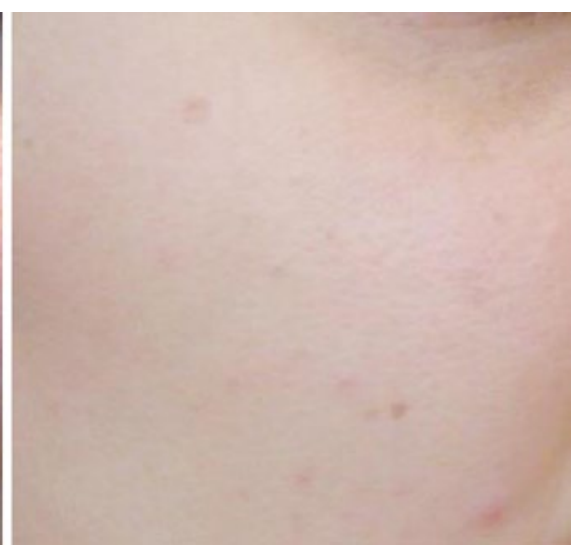

chronischen Wunden nach schweren Verbrennungen bringt. Die Anwendung von SHR-US demonstrierte auch schnelle und reproduzierbare Hautbildverbesserungen bei Ekzemen, perioraler Dermatitis und Psoriasis (• Abb. 5).

\section{Ästhetik}

Die Anti-Aging-Wirkung von SHF-US wurde in einer pragmatischen multizentrischen Studie an 39 Patienten geprüft [14]. Gesichtsbehandlungen wurden mit dualen US-Wellen $\left(\mathrm{LDM}^{\oplus}\right) 3 / 10 \mathrm{MHz}$ durchgeführt. Die Ergebnisse wurden von 4 unabhängigen Ärzten ausgewer- tet. Alle untersuchten Hautmerkmale (Falten, Hautporen und Hautturgor) waren nach der Behandlungsserie von 8 bis 12 Behandlungen signifikant verbessert. Der Placeboeffekt der Behandlung wurde durch kontralaterale Gesichtskontrolle ausgeschlossen. Die Zufriedenheit von Patienten und Ärzten auf einer 10-Stufen-Skala betrug jeweils $6,87 \pm 1,68$ und $7,23 \pm 1,62$. Die $\bullet$ Abb. 6 zeigt ein Beispiel der Hautbildverbesserung bei einer Patientin mit kontralateraler Kontrolle. Die - Abb. 7 demonstriert die Hautbildverbesserung nach 12 Tagen Behandlung mit 10-MHz-SHF-US

\section{) SHF-US kann auch supportiv zu anderen ästhetischen Therapiemethoden eingesetzt werden}

SHF-US kann nicht nur als selbstständige Methode, sondern auch supportiv zu anderen ästhetischen Therapiemethoden wie Laser und Radiofrequenz [5], Vakuummassage bei Body Contouring [9], Injektionslipolyse [26], Kryolipolyse [22], nach Schweißdrüsenkürettage bei Hyperhidrose [30] usw. eingesetzt werden. In allen diesen Studien und in mehreren Fallberichten wurde gezeigt, dass die Vorbehandlung mit SHF-US die klinischen Ergebnisse der Hauptmethode deutlich verbessert, während die Nachbehandlungen mit SHF-US die Entzündungsreaktionen, Schmerzen und die Rekonvaleszenzzeiten signifikant reduzieren.

\section{Fazit für die Praxis}

- SHF-US in Form von mono- oder dualfrequenten Wellen zeigt in der Dermatologie als auch in der Ästhetik gut reproduzierbare Behandlungsergebnisse $u$. a. bei verschiedenen entzündlichen Hauterkrankungen, chronischen Wunden und hypertrophen Narben sowie bei einigen wichtigen ästhetischen Indikationen, wie z. B. Anti-Aging oder allgemeine Hautbildverbesserung.

- Diese Methode ist schmerzfrei und nebenwirkungsarm und wird dadurch von Patienten sehr gut angenommen. 


\section{- Zusätzlich kann die dual-frequen- te Therapie $\left(\mathrm{LDM}^{\oplus}\right)$ auf der Basis von SHF-US als supportive Behandlungs- methode bei verschiedenen Licht- und Radiofrequenztherapieverfah- ren, bei Injektionslipolyse, bei ver- schiedenen Augmentationsbehand- lungen sowie in der ästhetisch-plasti- schen Chirurgie zur Vorbereitung und Nachsorge eingesetzt werden. Dabei können die Behandlungsergebnisse nicht nur signifikant verbessert, son- dern die auftretenden Nebenwirkun- gen sowie die Rekonvaleszenzzeiten erheblich reduziert werden.}

\section{Korrespondenzadresse}

Dr. rer. nat. habil. I. Kruglikov

Wellcomet $\mathrm{GmbH}$

Greschbachstr. 2-4

76229 Karlsruhe

i.kruglikov@wellcomet.de

\section{Einhaltung ethischer Richtlinien}

Interessenkonflikt. I. Kruglikov ist der geschäftsführende Gesellschafter der Firma Wellcomet $\mathrm{GmbH}$.

Dieser Beitrag beinhaltet keine Studien an Menschen oder Tieren.

Open Access Dieser Artikel unterliegt den Bedingungen der Creative Commons Attribution License. Dadurch sind die Nutzung, Verteilung und Reproduktion erlaubt, sofern der/die Originalautor/ en und die Quelle angegeben sind.

\section{Literatur}

1. Atalay M, Oksala N, Lappalainen J et al (2009) Heat shock proteins in diabetes and wound healing. Cur Prot Pept Sci 10:85-95

2. Bommannan D, Okuyama H, Stauffer P et al (1992) Sonophoresis. I. The use of high-frequency ultrasound to enhance transdermal drug delivery. Pharm Res 9:559-564

3. Bommannan D, Menon GK, Okuyama $\mathrm{H}$ et al (1992) Sonophoresis. II. Examination of the mechanism(s) of ultrasound-enhanced transdermal drug delivery. Pharm Res 9:1043-1047

4. Emanuele E, Bertona M, Altabas K et al (2012) Anti-inflammatory effects of topical preparation containing nicotinamide, retinol, and 7-dehydrocholesterol in patients with acne: a gene expression study. Clin Cosmet Investig Dermatol 5:33-37

5. Gansel R (2015) Kombinationsverfahren mit Hautbildverbesserung. Ästhet Dermatol. (To be published)

6. Gohla T, Kruglikova E, Kruglikov I (2012) Treatment of non-healing, post-traumatic wound with high frequency ultrasound of $10 \mathrm{MHz}$ : A case report. $\mathrm{Br}$ J Med Med Res 2:520-526
7. Kang S, Cho S, Chung JH et al (2005) Inflammation and extracellular matrix degradation mediated by activated transcription factors nuclear fact- $\mathrm{kB}$ and activator protein-1 in inflammatory acne lesions in vivo. Am J Pathol 166:1691-1699

8. Kang KS, Hong JM, Kang JA, et al (2013) Osteogenic differentiation of human adipose-derived stem cells can be accelerated by controlling the frequency of continuous ultrasound. J Ultrasound Med 32:1461-1470

9. Kruglikov I (2012) Biophysical basics of body treatments: a preliminary study into the correlation between washout and circumference reduction. Am J Cosm Surg 29:196-202

10. Kruglikov I, Sontag W (2010) Ultrasound of $10 \mathrm{MHz}$ frequency as a novel strategy for skin anti-aging therapy. Med Hypotheses 74:620-621

11. Kruglikov IL, Kruglikova E (2011) Dual treatment strategy by venous ulcers: pilot study to dual-frequency ultrasound application. J Cosm Dermatol Sci Appl 1:157-163

12. McLennan SV, Min D, Yue DK (2008) Matrix metalloproteinases and their roles in poor wound healing in diabetes. Wound Pract Res 16:116-121

13. Meyer-Rogge D, Kruglikov I (2013) Pilot study into super-fractionation treatment strategy of acne and rosacea. J Cosm Dermatol Sci Appl 3:197-202

14. Meyer-Rogge D, Rösken F, Holzschuh P et al (2012) Facial skin rejuvenation with high frequency ultrasound: multicentre study of dual-frequency ultrasound. J Cosm Dermatol Sci Appl 2:68-73

15. Miller DL (2007). Overview of experimental studies of biological effects of medical ultrasound caused by gas body activation and inertial cavitation. Prog Biophys Mol Biol 93:314-330

16. Morris SD (2002) Heat shock proteins and the skin. Clin Exp Dermatol 27:220-224

17. Oberringer M, Baum HP, Jung V et al (1995) Differential expression of heat shock protein 70 in well healing and chronik human wound tissue. Biochem Biophys Res Commun 214:1009-1014

18. Polat BE, Hart D, Robert Langer R et al (2011) Ultrasound-mediated transdermal drug delivery: Mechanisms, scope, and emerging trends. J Control Release 152:330-348

19. Purwar R, Kraus M, Werfel T et al (2008) Modulation of keratinocyte-derived MMP-9 by IL-13: a possible role for the pathogenesis of epidermal inflammation. J Invest Dermatol 128:59-66

20. Reiss MJ, Han YP, Garcia E et al (2010) Matrix metalloproteinase- 9 delays wound healing in a murine wound model. Surgery 147:295-302

21. Reno F, Grazianetti P, Stella M et al (2002) Release and activation of matrix metalloproteinase- 9 during in vitro mechanical compression in hypertrophic scars. Arch Dermatol 138:475-478

22. Sandhofer M (2013) Zur Kryolipolyse in der dermatologischen Praxis. Kosmet Med 34:100-109

23. Schmidt BA, Horsley V (2013) Intradermal adipocytes mediate fibroblast recruitment during skin wound healing. Develop 140:1517-1527

24. Sontag W, Kruglikov I (2009) Expression of heat shock proteins after ultrasound exposure in $\mathrm{HL}-60$ Cells. Ultra Med Biol 35:1032-1041

25. Tanriverdi-Akhisaroglu S, Menderes A, Oktay G (2009) Matrix metalloproteinase-2 and -9 activities in human keloids, hypertrophic and atrophic scars: a pilot study. Cell Biochem Func 27:81-87

26. Tausch I, Kruglikov I (2015) The benefit of supportive application of dual-frequency ultrasound in patients treated by injection lipolysis. J Clin Aesth Dermatol 8:20-24
27. Totan S, Echo A, Yuksel E (2011) Heat shock proteins modulate keloid formation. Eplasty 11:190 202

28. Uhlemann C, Wollina U (2003) Wirkungsphysiologische Aspekte des therapeutischen Ultraschalls in der Wundbehandlung. Phlebol 32:81-85

29. Weichenthal M, Mohr P, Stegman W et al (1997) Low-Frequency ultrasound treatment of chronic venous ulcers. Wound Rep Regen 5:18-22

30. Weidmann M (2015) Application of dual-frequency ultrasound improves the induration by patients with axillary hyperhidrosis treated by suction-curettage. J Clin Aesth Dermatol. (To be published)

31. WegrowskiY (1993) Effect of hyperthermia on the extracellular matrix. I. Heat enhances hyaluronan and inhibits sulphated glycosaminoglycan synthesis. FEBS Lett 334:121-124 